\title{
The pattern of epidermal growth factor receptor variation with disease progression and aggressiveness in colorectal cancer depends on tumor location
}

\author{
PETROS C. PAPAGIORGIS ${ }^{1}$, ADAMANTIA E. ZIZI ${ }^{2}$, SOPHIA TSELENI ${ }^{3}$, \\ IOANNIS N. OIKONOMAKIS ${ }^{4}$ and NIKOLAOS I. NIKITEAS ${ }^{5}$
}

${ }^{1}$ Department of Surgery, Athens Medical Center, Marousi $15125 ;{ }^{2}$ Department of Pathology, Tzaneio General Hospital,
Piraeus $18536 ;{ }^{3}$ Department of Pathology, Medical School, University of Athens, Athens $11527 ;$
${ }^{4}$ Department of Surgery, 401 Army General Hospital, Athens; ${ }^{5}$ Second Department of Propedeutic Surgery,
Medical School, University of Athens, Athens 11527 , Greece

Received October 20, 2011; Accepted January 12, 2012

DOI: $10.3892 / \mathrm{ol} .2012 .621$

\begin{abstract}
The role of epidermal growth factor receptor (EGFR) in colorectal cancer (CRC) prognosis remains unclear despite the recent development of anti-EGFR treatments for metastatic disease. The heterogeneity of CRC may account for this discrepancy; proximal and distal CRC has been found to be genetically and clinicopathologically different. The aim of this study was to investigate the effect of tumor location on the association of EGFR with the conventional prognostic indicators (stage and grade) in CRC. Immunohistochemical assessment of EGFR was retrospectively performed in 119 primary CRC specimens and data were correlated with tumor stage and grade in the proximal and distal tumor subset. The molecular combination of EGFR with p53 (previously assessed in this sample) was similarly analyzed. EGFR positivity was detected in 34, 30 and $35 \%$ of the entire cohort, proximal and distal tumors, respectively. The pattern of EGFR clinicopathological correlation was found to differ by site. A reduction in the frequency of $\operatorname{EGFR}(+)$ with progression of stage and/or worsening of grade was observed proximally, whereas an opposite trend was recorded distally. Proximal tumors with stage I or with indolent features (stage I, welldifferentiated) exhibited a significantly higher proportion of EGFR positivity than other tumors of this location $(\mathrm{p}=0.023$
\end{abstract}

Correspondence to: Dr Petros C. Papagiorgis, Department of Surgery, Athens Medical Center, Distomou 5-7, Marousi 151 25, Greece

E-mail: ppapagiorg@teiath.gr

Abbreviations: EGFR, epidermal growth factor receptor; CRC, colorectal cancer; G1, Grade 1; G2, Grade 2; G3, Grade 3; MSI, microsatellite instability; CIMP, $\mathrm{CpG}$ island methylator phenotype; CIN, chromosomal instability

Key words: proximal and distal colorectal cancer, epidermal growth factor receptor, stage, grade and $\mathrm{p}=0.022$, respectively) or corresponding distal tumors $(\mathrm{p}=0.018$ and $\mathrm{p}=0.035$, respectively). Moreover, the co-existence of EGFR and high p53 staining (accounting for 11\% of cases) was found in a significantly higher proportion of stage IV tumors compared to other stages $(\mathrm{p}=0.004)$, although only for the distal subset. Proximal and distal tumors showed various patterns of EGFR variation with disease progression and aggressiveness. This disparity provides further support to the hypothesis that these particular subsets of CRC are distinct tumor entities. It may also be suggestive of a potentially different therapeutic approach according to tumor site, particularly regarding anti-EGFR targeted treatment.

\section{Introduction}

Colorectal cancer (CRC) is one of the most common malignancies and remains a major cause of cancer mortality in the West (1). It is also a multi-pathway disease with disparate subgroups exhibiting distinct genetic and clinicopathological features, and probably different outcomes (2). This may be the main reason for the variability in treatment response observed among patients of the same disease stage. Therefore, a combination of the conventional TNM staging classification (at present, the major prognostic indicator) with certain molecular markers involved in CRC tumorigenesis, with verified prognostic and predictive impact, is one of the main objectives of research worldwide (3).

Epidermal growth factor receptor (EGFR) is a transmembrane glycoprotein member of the tyrosine-kinase receptor family, encoded by the c-erB1 proto-oncogene and is considered as a major regulator of several distinct cellular pathways. Activation of EGFR promotes carcinogenesis, by increasing proliferation, cell migration, angiogenesis and apoptosis inhibition (4-6). On this basis, targeted therapies using anti-EGFR antibodies and tyrosine-kinase inhibitors are now an approved treatment in metastatic CRC $(7,8)$. However, immunohistochemically assessed EGFR expression has not been validated as a predictor of response to this specific treatment. Moreover, the impact of EGFR expression on the outcome of CRC 
patients is generally unclear (3). Methodological variability, indicated by the wide range (18-97\%) in the detected frequencies of EGFR in CRC (9-14), may be responsible for this effect.

Heterogeneity of CRC (2) should also be taken into account since EGFR expression may be discordant among primary tumors, lymph nodes and metastases $(10,11)$. It may also be related to tumor stage and grade, although the reported results on this issue are inconsistent (9-14). However, there has been limited attention regarding the association of EGFR with tumor site, despite the considerable molecular and clinicopathological differences between proximal (right-sided) and distal (left-sided) CRC (15-18), suggesting the existence of two distinct disease entities with different outcomes and treatment responses $(19,20)$.

In this study, differences regarding the immunohistochemically assessed EGFR expression rate were examined in a series of CRC cases previously investigated for segmental differences in other molecular markers (18). We analyzed the correlation of EGFR with stage and grade (i.e., the conventional prognostic indicators) in the entire cohort and in the proximal and distal tumor subsets. We also examined the correlation between EGFR and the previously assessed p53 (18), considering the central tumorigenic role of the latter marker along with its known predilection for the distal tumor site $(2,15,16,18)$.

\section{Materials and methods}

Study population. Hospital records of 147 unselected cases that underwent surgery for CRC between 2000 and 2003 in the Second Surgical Department of Tzaneio Hospital of Piraeus were retrospectively examined. Following the omission of recurrences, hereditary cases, synchronous cancers of double location and those with unclear pathology reports or insufficient tissue for analysis, 119 patients (69 males, 50 females; mean age, 69.3 years; range, 32-90 years) were included in the study, providing a homogenous sample of primary, sporadic and untreated cases. None of the cases had undergone neoadjuvant therapy, as it was not performed during the selected study period at this hospital. The study was approved by the Surgical department of the Athens Medical School.

Immunohistochemistry. Sections $(5 \mu \mathrm{m})$ were obtained from paraffin-embedded tissue blocks of primary tumor specimens. The immunoperoxidase method was performed in three steps, using an Envision Dako kit (Glostrup, Denmark). EGFR was assessed with anti-EGFR mouse monoclonal antibody (dilution 1:200, Dako). Diaminobenzidine (DAB, 0.6\%) was used as a chromogen and tissues were counter-stained with hematoxylin. Normal epidermis with a known EGFR status served as a positive control, whereas pre-immune rabbit serum was used as a negative control.

Staining interpretation. Immunoreactivity was independently evaluated by two observers (blinded to clinicopathological information) and discrepancies between them were resolved by consensus. Any lesion with distinctly visible staining [membranous and/or cytoplasmic $(9,11,12)]$ was considered positive.

Multiple cutoffs and the scoring of staining intensity (a less objective criterion), or complex scoring (i.e., combining percentages with intensity) were avoided as they all potentially increase interobserver variability (21). Moreover, multiple stratification, used for other markers (including p53) in our previous study (18), was inappropriate due to the relatively low proportion of EGFR positivity (see Results). The selected threshold was similar to that used in previous studies (1\%), revealing strong prognostic and clinicopathological correlations of EGFR $(10,12,13)$.

Clinicopathological classification. Cases were classified according to the results of their pathology report as stage I, II, III or IV using the TNM classification, and Grade 1 (G1, welldifferentiated), 2 (G2, moderately differentiated) or 3 (G3, poorly differentiated) using the WHO classification. The cases were also classified by site, as proximal (cecum, ascending, transverse) and distal (descending, sigmoid, rectum), in relation to the splenic flexure (15-18).

Moreover, considering the small size of certain subsets and the fact that we aimed to examine the combined effect of stage and grade on EGFR distribution, we stratified tumors into three additional categories, modifying the corresponding classification previously implemented by Resnic et al (14): i) cases with at least one indolent feature (stage I or G1), ii) cases with at least one unfavorable feature (stage IV or G3) and iii) cases with intermediate tumor characteristics (stages II-III with moderate grade). Given the absence of tumors with completely conflicting features (stage I/G3 or IV/G1) in our sample, there was no need for exclusion of such cases.

Statistical analysis. The distribution of EGFR expression among various clinicopathological variables was analyzed using the $\chi^{2}$ test (with Yates correction when necessary) and Fisher's exact test (appropriate for categorical comparisons between small subsets). EGFR distribution by stage and grade (or their combination) was separately examined in the proximal and distal subsets using the same tests. Moreover, on the basis of the previously recorded data for p53 (18), the distribution of the various molecular combinations between EGFR and p53 was similarly analyzed, particularly focusing on the pattern of tumors combining EGFR with a high p53 expression level $(>60 \%)$. Tests were two-sided, with $p$ values $\leq 0.05$ considered to indicate a statistically significant difference.

\section{Results}

Clinicopathological parameters and immunohistochemistry. Table I shows that moderate grade $(86.5 \%)$, stage II-III (79\%) and distal tumor location (70\%) were the prevailing features in this sample. Positive EGFR expression was detected in 40 cases (34\%), with typical immunostaining shown in Fig. 1. EGFR positivity was almost uniformly distributed among the various clinicopathological subsets. Even for tumor grade, the apparently considerable variation of EGFR was not significant. No association was observed between EGFR positivity and the previously assessed (18) high p53 staining found in $25 \%$ of cases.

Pattern of staining variation by stage and grade was different for each segment. EGFR expression was slightly higher in the distal compared to the proximal site tumors (35 vs. $30.5 \%$ ), but the difference was insignificant (Table I). However, the 
Table I. Clinicopathological and immunohistochemical features.

\begin{tabular}{|c|c|c|c|c|c|}
\hline \multirow[t]{2}{*}{ Parameters } & \multicolumn{2}{|c|}{ Cases } & \multicolumn{2}{|c|}{$\operatorname{EGFR}(+)$} & \multirow[t]{2}{*}{ P-value } \\
\hline & $\mathrm{n}$ & $(\%)^{\mathrm{a}}$ & $\mathrm{n}$ & $(\%)^{\mathrm{b}}$ & \\
\hline TNM stage & & & & & NS \\
\hline I & 12 & (10) & 4 & (33) & \\
\hline II & 50 & $(42)$ & 16 & $(32)$ & \\
\hline III & 44 & (37) & 16 & $(36)$ & \\
\hline IV & 13 & $(11)$ & 4 & (31) & \\
\hline Grade & & & & & NS \\
\hline Well (G1) & 7 & $(6)$ & 4 & (57) & \\
\hline Moderate (G2) & 103 & $(86.5)$ & 35 & (34) & \\
\hline Poor (G3) & 9 & $(7.5)$ & 1 & $(11)$ & \\
\hline Combined stage-grade & & & & & NS \\
\hline Indolent $^{\mathrm{c}}$ & 16 & $(13.5)$ & 6 & $(37.5)$ & \\
\hline Intermediate $^{\mathrm{d}}$ & 82 & $(69)$ & 29 & $(35)$ & \\
\hline Unfavorable $^{\mathrm{e}}$ & 21 & $(17.5)$ & 5 & (24) & \\
\hline Tumor site & & & & & NS \\
\hline Proximal (right) & 36 & $(30)$ & 11 & $(30)$ & \\
\hline Distal (left) & 83 & $(70)$ & 29 & $(35)$ & \\
\hline Gender & & & & & NS \\
\hline Male & 69 & (58) & 25 & (36) & \\
\hline Female & 50 & $(42)$ & 15 & $(30)$ & \\
\hline Age & & & & & NS \\
\hline$<70$ & 56 & (47) & 19 & (34) & \\
\hline$>70$ & 63 & $(53)$ & 21 & (33) & \\
\hline p53 staining & & & & & NS \\
\hline $\operatorname{High}^{\mathrm{f}}$ & 30 & $(25)$ & 13 & (43) & \\
\hline Low $^{\mathrm{g}}$ & 89 & (75) & 27 & (30) & \\
\hline Total & 119 & $(100)$ & 40 & (34) & \\
\hline
\end{tabular}

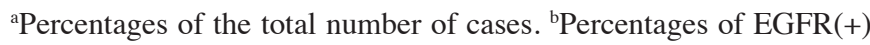

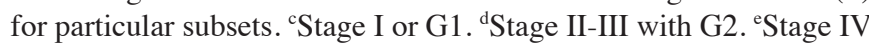

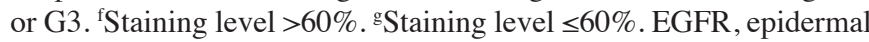
growth factor receptor. NS, not significant.

observed pattern of staining variation by stage and grade was markedly different for each particular segment (Table II); the frequency of EGFR in the proximal subset varied between $100 \%$ (stage I) and 0\% (stage IV). Conversely, EGFR frequencies ranging from 11 (stage I) to 50\% (stage IV) were observed distally. Subset analysis revealed: i) a significant difference of EGFR expression frequencies between stage I and the other stages (II, III and IV) of the proximal segment considered together $(\mathrm{p}=0.023)$ or separately $(\mathrm{p}=0.04,0.07,0.02$, respectively); and ii) a significant difference of the EGFR staining frequency between proximal and distal tumors with stage I disease $(\mathrm{p}=0.018)$, whereas the corresponding segmental difference for stage IV did not reach the level of significance ( $\mathrm{p}=0.1)$ (Table II, Fig. 2).

Moreover, a progressive reduction in EGFR frequency was observed with worsening of grade. This pattern was recorded for the overall series (from $57 \%$ in Grade 1 to $11 \%$ in Grade 3)

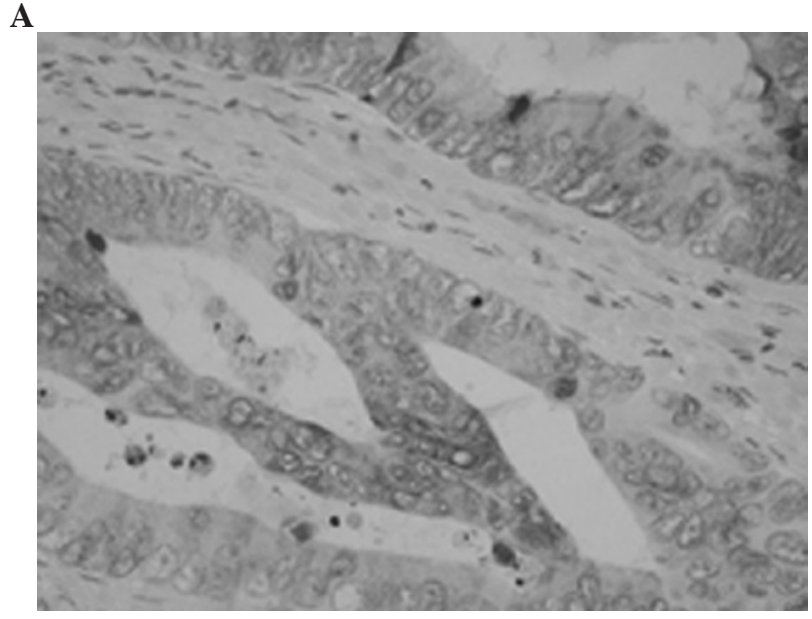

B

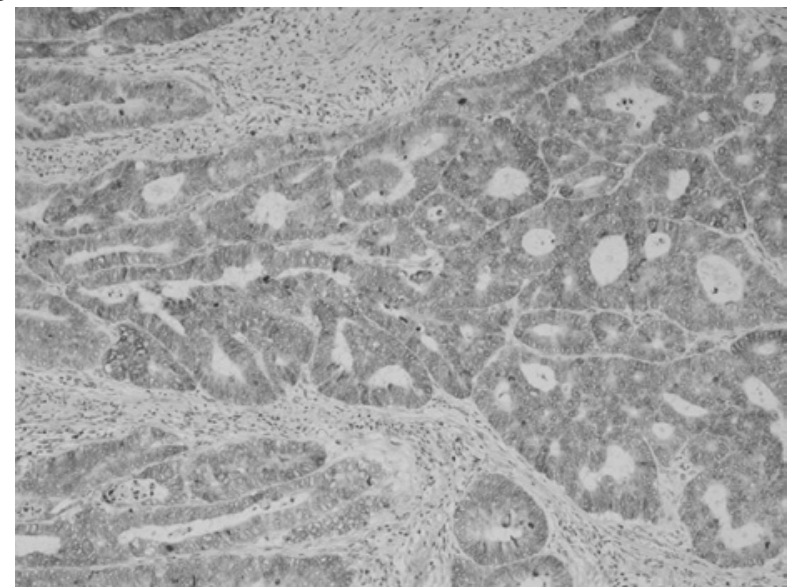

Figure 1. EGFR immunostaining. (A and B) Colorectal cancer specimens with representative immunostaining positive for EGFR monoclonal antibody (magnification, x200). EGFR, epidermal growth factor receptor.

and for the proximal subset (from $75 \%$ in Grade 1 to $14 \%$ in Grade 3) but was somewhat modified in the distal subset (Table II, Fig. 3). However, the observed differences of EGFR staining between particular grades (of the same segment), or between proximal and distal tumors of the same grade were not significant, although they approached the level of significance in certain comparisons within the proximal subset (G1 vs. G2-G3, p=0.075 and G1 vs. G3, p=0.09).

EGFR expression was examined in three additional tumor subsets including tumors with indolent (stage I or G1, 16 cases), unfavorable (stage IV or G3, 21 cases) and intermediate (stage II-III with moderate grade, 82 cases) clinicopathological features. The results of this analysis were similar to the previously ascertained findings regarding EGFR stage distribution; the indolent subset exhibited a significantly higher proportion of EGFR positivity compared with the other subsets (80 vs. $22 \%, p=0.022$ ), although only for proximal cases. Additionally, the frequency of $\operatorname{EGFR}(+)$ was significantly higher proximally than distally for the indolent cases ( 80 vs. $37.5 \%, p=0.035$; Table II and Fig. 4).

Molecular combinations were significantly elevated in stage $I V$. Although the tumor site was found to be unrelated to any molecular combination of EFGR with p53 (Table III), 
Table II. EGFR segmental distribution by stage and grade.

\begin{tabular}{|c|c|c|c|c|c|}
\hline & \multicolumn{2}{|c|}{ Proximal } & \multicolumn{2}{|c|}{ Distal } & \multirow[t]{2}{*}{ P-value ${ }^{a}$} \\
\hline & $\mathrm{n}$ & $\operatorname{EGFR}(+)(\%)$ & $\mathrm{n}$ & $\operatorname{EGFR}(+)(\%)$ & \\
\hline \multicolumn{6}{|l|}{ Stage } \\
\hline $\mathrm{I}$ & 3 & $3(100)$ & 9 & 1 (11) & 0.018 \\
\hline II & 13 & $3(23)$ & 37 & $13(35)$ & NS \\
\hline III & 15 & $5(33)$ & 29 & $11(38)$ & NS \\
\hline IV & 5 & $-(0)$ & 8 & $4(50)$ & 0.1 \\
\hline \multicolumn{6}{|l|}{ Grade } \\
\hline Well (G1) & 4 & $3(75)$ & 3 & $1(33)$ & NS \\
\hline Moderate (G2) & 25 & $7(28)$ & 78 & $28(36)$ & NS \\
\hline Poor (G3) & 7 & 1 (14) & 2 & - (0) & NS \\
\hline \multicolumn{6}{|c|}{ Combined stage - grade } \\
\hline Indolent $^{\mathrm{b}}$ & 5 & $4(80)$ & 11 & $2(18)$ & 0.035 \\
\hline Intermediate $^{\mathrm{c}}$ & 20 & $6(30)$ & 62 & $23(37)$ & NS \\
\hline Unfavorable $^{\mathrm{d}}$ & 11 & $1(9)$ & 10 & $4(40)$ & $\mathrm{NS}(0.12)$ \\
\hline Total & 36 & $11(30.5)$ & 83 & $29(35)$ & NS \\
\hline
\end{tabular}

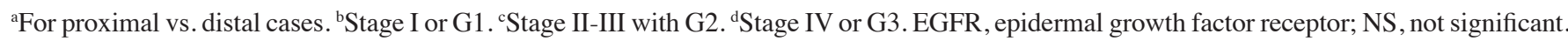

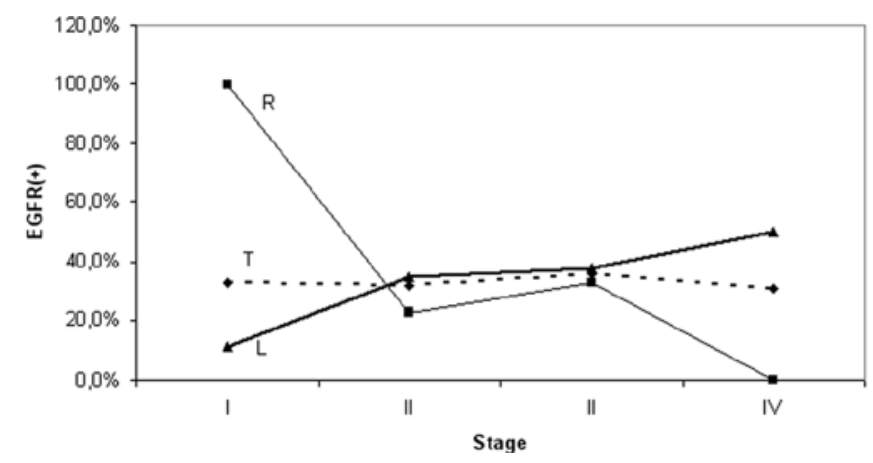

Figure 2. Variation of EGFR with stage. The frequency of EGFR positivity was reduced with disease progression for right-sided tumors $(\mathrm{R})$. An opposite trend was shown by left-sided lesions (L). As a result, EGFR positivity was evenly distributed among stages of the total sample (T). Significant p-values of EGFR variation were recorded for right-sided stage I vs. other stages considered together (I vs. II-IV, p=0.023) or separately (I vs. II, III, IV/p=0.04, 0.07, 0.02 , respectively). EGFR, epidermal growth factor receptor.

cases with EGFR(+)/p53 high immunoreactivity (accounting for $11 \%$ of the total sample) were more frequently detected in stage IV than in other stages ( 31 vs. $8.5 \%, \mathrm{p}=0.051$ ). This trend was stronger and significant for distal tumors (50 vs. $8 \%$, $\mathrm{p}=0.004$ ) and completely absent for corresponding proximal tumors (Fig. 5).

\section{Discussion}

The involvement of EGFR activation in a number of cellular pathways promoting tumorigenesis may explain the benefit from the recently implemented anti-EGFR therapies (i.e., cetuximab or panitumab) $(3,7,8)$. Nonetheless, the prognostic and predictive value of EGFR status in CRC remains uncertain (3). However, the effect of EGFR on prognosis and

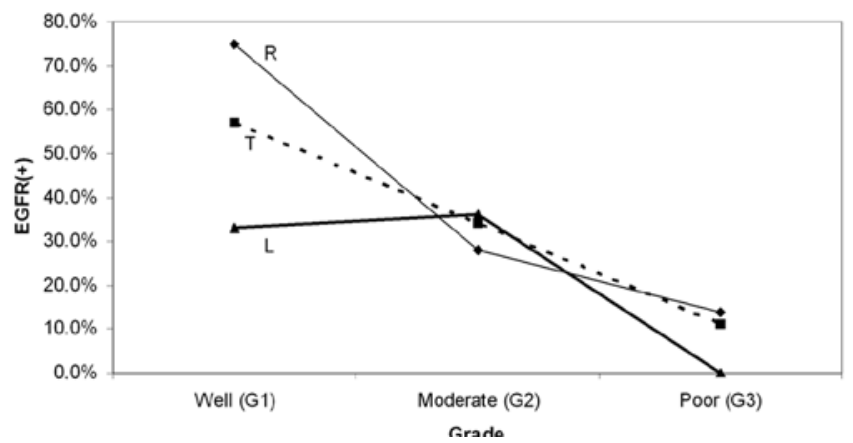

Figure 3. Variation of EGFR with grade. A progressive reduction of EGFR(+) cases with worsening of tumor grade is shown for the total sample $(\mathrm{T})$ and the right-sided subset $(\mathrm{R})$, with the two groups showing similar variation patterns. The corresponding pattern for left-sided tumors (L) was somewhat differential. None of the observed differences of EGFR positivity, between right and left subsets or between particular grades (G1, G2, G3) of the same category $(\mathrm{R}, \mathrm{L}, \mathrm{T})$ were significant. However, the trend for EGFR(+) shown by the well-differentiated tumors approached significance for the right subset (G1 vs. G2-G3, p=0.075 and G1 vs. G3, p=0.09) and the total sample (G1 vs. $\mathrm{G} 3, \mathrm{p}=0.08)$. EGFR, epidermal growth factor receptor.

treatment response may vary among genetically different tumors; proximal and distal CRC have been considered to evolve through different genetic pathways [microsatellite instability/CpG island methylator phenotype (MSI/CIMP) and chromosomal instability (CIN), respectively] $(15,16)$ with disparate clinicopathological features (17-19) and possibly different outcomes $(19,20)$.

In the current study, we examined the impact of tumor site on EGFR distribution in particular clinicopathological variables. The observed variation in EGFR detection rate with disease progression (from stage I to IV) was found to differ between proximal and distal tumors, showing a reduction 
Table III. Molecular combinations between EGFR and p53.

\begin{tabular}{|c|c|c|c|c|c|c|c|}
\hline & \multicolumn{2}{|c|}{ Total } & \multicolumn{2}{|c|}{ Proximal } & \multicolumn{2}{|c|}{ Distal } & \multirow[t]{2}{*}{ P-value } \\
\hline & $\mathrm{n}$ & $\%$ & $\mathrm{n}$ & $\%$ & $\mathrm{n}$ & $\%$ & \\
\hline \multicolumn{8}{|l|}{ Marker } \\
\hline $\operatorname{EGFR}(+)$ & 40 & (34) & 11 & $(30)$ & 29 & (35) & NS \\
\hline p53 high ${ }^{\mathrm{b}}$ & 30 & $(25)$ & 7 & $(19.5)$ & 23 & (28) & NS \\
\hline \multicolumn{8}{|l|}{ Marker combination } \\
\hline EGFR(+)/p53 high ${ }^{\mathrm{b}}$ & 13 & (11) & 3 & (8) & 10 & (12) & NS \\
\hline $\operatorname{EGFR}(-) / p 53$ low $^{\mathrm{c}}$ & 62 & $(52)$ & 21 & $(58)$ & 41 & $(50)$ & NS \\
\hline EGFR(-)/p53 high ${ }^{b}$ & 17 & (14) & 4 & (11) & 13 & $(15.5)$ & NS \\
\hline EGFR(+)/p53 low & 27 & $(22.5)$ & 8 & $(22)$ & 19 & $(23)$ & NS \\
\hline Total & 119 & $(100)$ & 36 & $(100)$ & 83 & $(100)$ & \\
\hline
\end{tabular}

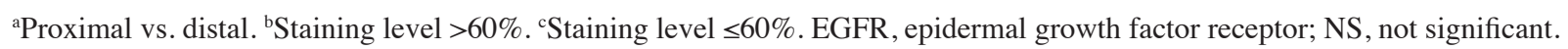

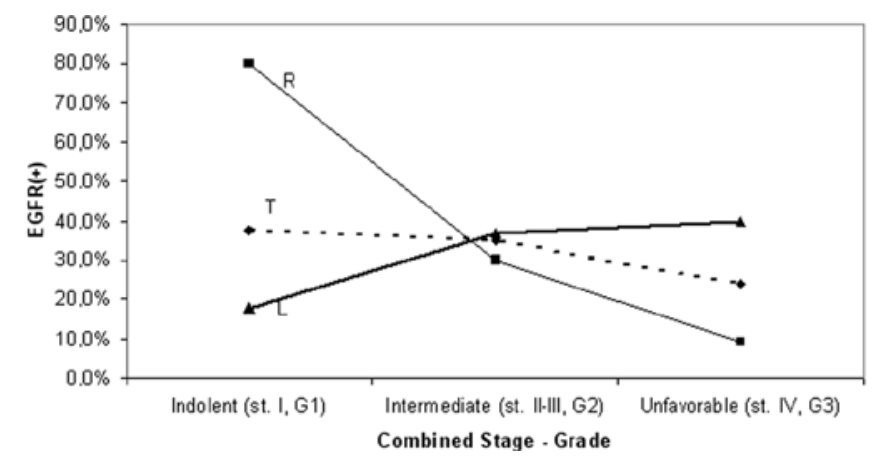

Figure 4. Variation of EGFR according to combined tumor characteristics (stage-grade). The pattern of EGFR variation with disease change from indolent to unfavorable was different for the right- (R) and the left- (L) sided lesions, revealing reductions and elevations of the EGFR detection rate, respectively. Corresponding EGFR variation in the total sample (T) was limited. Significant p-values were found for the observed variation in rightsided tumors (indolent vs. others, $\mathrm{p}=0.022$ and indolent vs. unfavorable, $\mathrm{p}=0.013)$. EGFR, epidermal growth factor receptor.

and elevation of this rate, respectively. Proximal lesions also showed a similar decrease in the proportion of EGFR positivity with worsening of grade and, as expected, with the change of the combination of stage and grade from indolent to unfavorable. By contrast, for distally located tumors, the same change appeared to have the opposite effect (elevation).

Notably, these trends were not present in the entire cohort, with the exception of EGFR variation by grade, consistent with previous results (11). This lack of EGFR correlation with stage and grade has been also reported by other authors $(9,14,22)$, whereas inconsistent findings are presented among studies suggesting such connections $(10,12,13)$. Therefore, a separate investigation of proximal and distal CRC appears to be necessary for a more accurate determination of the effect of EGFR status on the progression, aggressiveness and, probably, the outcome of the disease. In this respect, the fact that EGFR status has failed to predict response in metastatic cases that underwent anti-EGFR therapy may be partially explained by the observed rarity of EGFR positivity in proximal metastatic

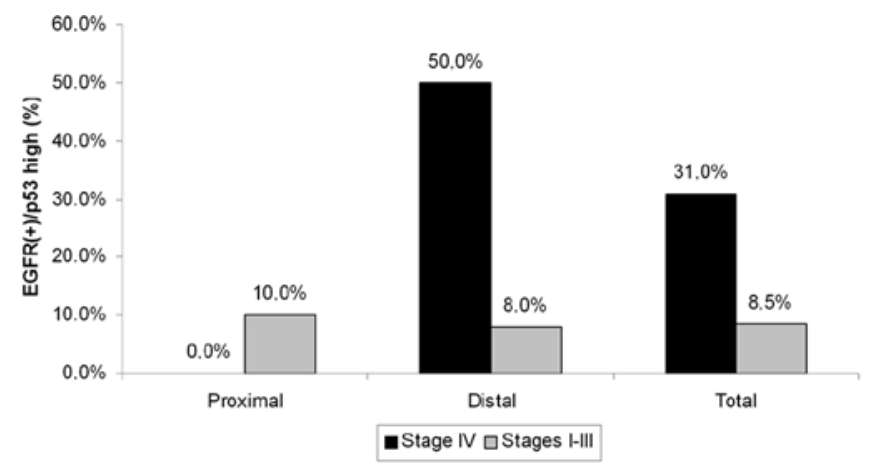

Figure 5. Variation of EGFR(+)/p53 high combination with stage. The frequency of the EGFR(+)/p53 high molecular combination was significantly higher in stage IV than in the other stages (I-III) for the total sample $(\mathrm{p}=0.051)$ and particularly for distal tumors $(\mathrm{p}=0.004)$. However, such a correlation was not observed in proximal tumors (actually revealing a lack of this combination among stage IV cases). EGFR, epidermal growth factor receptor.

disease. Corresponding rarity of $\operatorname{EGFR}(+)$ in cases with poor grade may also be relevant (11); aggressive lesions are more commonly found in advanced stages, as well as at the proximal site (23).

Moreover, tumors with the EGFR(+)/p53 high molecular combination, exhibited a predilection for stage IV, which was particularly pronounced in the distal subset. This observation may be explained by the reported connection of p53 inactivation with both distal site $(15,18,24)$ and higher stage $(24)$, particularly stage IV (25). Nevertheless, the observed trend for metastatic disease (if validated) could be clinically useful, facilitating the selection of cases for chemotherapy and/or anti-EGFR therapy, based on combined EGFR/p53 status and tumor location. Notably, as recently reported, p53 mutation may predict response to cetuximab treatment (26), suggesting that p53 inactivation is likely one of the mechanisms leading to EGFR activation, as indicated by the $90 \%$ concordance between p53 mutations and the EGFR copy number increase (26). 
Given that, at present, sufficient evidence of prognostic and predictive significance for any single marker is lacking, including EGFR and p53 $(3,27)$, the potential usefulness of marker combinations appears to be a more promising approach $(28,29)$. In this context, and as regards EGFR, it has recently been reported that the effectiveness of anti-EGFR therapy in metastatic CRC is decreased in cases with $\mathrm{Ki}$-Ras (30) or BRAF and PTEN mutations (31). The impact of the tumor site on these findings should also be examined, considering reported associations of $\mathrm{Ki}$-Ras mutations with metastatic disease and worse outcome, particularly detected in distal tumors $(32,33)$.

A limitation of our study is that the main findings were detected in small subsets (stage I, IV - Grade 1, 3). However, despite the modest size of our sample, our results were similar to those of several relevant studies $(9,10,12-14,22)$. Although we confirmed these results in the expanded additional subsets [created by combining stage and grade: interrelated features differentially representing tumor growth potential (34)], further investigation in a larger sample is necessary. Another limitation is the long-standing filing of paraffin blocks (7-10 years), which is shown to reduce EGFR immunoreactivity (35). Such an effect may explain the decreased EGFR(+) detection rate in our sample (34\%) compared to those seen in other studies with similar thresholds $(12,22,36)$, ranging from 50 to $97 \%$. However, Galizia et al (13), using a similar cut-off value, found almost equal frequencies of EGFR $(+)(35 \%)$, whereas other authors $(10,37)$ reported even lower rates $(18$ and $21.5 \%$, respectively).

However, the simplicity of our methodology in EGFR staining interpretation minimizes interobserver variability, facilitates reproducibility and is appropriate for samples of this size, with an observed $\operatorname{EGFR}(+)$ detection rate (34\%). However, in larger samples, or in those with higher detection rates and a wide range in the observed percentages of positivity, the use of multiple thresholds or complicated scoring systems may provide better information (21).

Moreover, our data indicated the importance of separate segmental analysis in revealing clinicopathological correlations of EGFR; the uniform distribution of this marker among stages in the entire cohort was resulted (mostly) from the combined effect of the opposite trends in EGFR variation with disease progression recorded for proximal and distal subsets. Similarly, the counteraction between the indolent and the unfavorable tumor subsets, exhibiting different segmental predilections of EGFR positivity (for proximal and distal site, respectively), contributed to the observed lack of segmental difference in overall sample, perhaps explaining the reason for such differences having rarely been reported (38). Even more detailed analysis may be necessary; in particular, colon segments (cecum, ascending and sigmoid) have recently been found showing distinct clinicopathological features (39), possibly reflecting underlying molecular disparities.

In conclusion, in this exploratory study we found that the pattern of EGFR variation with disease progression and/or aggressiveness differed according to tumor location. Although these results support that proximal and distal CRC are different disease entities, their potential impact on prognosis and treatment should be investigated. Additional investigations may include: i) meta-analyses of selected EGFR immunohisto- chemical studies with, preferably, similar methodology; ii) large retrospective site-specific analyses of EGFR predictiveness in patients receiving anti-EGFR treatment; and iii) corresponding prospective studies. In future, therapy decisions may be based on the combined clinicopathological and molecular tumor status, possibly including EGFR and tumor site.

\section{Acknowledgements}

We thank Drs Th. Vlassis, F. Georgiadis and I. Elemenoglou for their help and Mrs. N. Vathi for valuable assistance in the preparation of the manuscript.

\section{References}

1. Jemal A, Siegel R, Ward E, et al: Cancer statistics. CA Cancer J Clin 58: 71-96, 2008.

2. Jass JR: Classification of colorectal cancer based on correlation of clinical, morphological and molecular features. Histopathology 50: 113-130, 2007.

3. Tejpar S: The use of molecular markers in the diagnosis and treatment of colorectal cancer. Best Pract Res Clin Gastroenterol 21: 1071-1087, 2007.

4. Zwick E, Hackel PO, Prenzel N and Ullrich A: The EGF receptor as central transducer of heterologous signaling systems. Trends Pharmacol Sci 20: 408-412, 1999.

5. Salomon D, Brandt R, Ciardiello F and Normanno N: Epidermal growth factor - related peptides and their receptors in human malignancies. Crit Rev Oncol Hematol 19: 183-232, 1995.

6. Klapper LN, Kirschbaum MH, Sela M and Yarden Y: Biochemical and clinical implications of the ErbB/HER signaling network of growth factor receptors. Adv Cancer Res 77: 25-79, 2000.

7. Tol J and Punt CJ: Monoclonal antibodies in the treatment of metastatic colon cancer: a review. Clin Ther 32: 437-453, 2010.

8. Modjtahedi $\mathrm{H}$ and Essapen S: Epidermal growth factor receptor inhibitors in cancer treatment: advances, challenges, and opportunities. Anticancer Drugs 20: 851-855, 2009.

9. Kountourakis P, Pavlakis K, Psyrri A, et al: Clinicopathologic significance of EGFR and Her-2/neu in colorectal adenocarcinomas. Cancer J 12: 229-236, 2006.

10. Deng Y, Kurland B, Wang J, et al: High epidermal growth factor receptor expression in metastatic colorectal cancer lymph nodes may be more prognostic of poor survival than in primary tumor. Am J Clin Oncol 32: 245-252, 2009.

11. McKay JA, Murray LJ, Curran S, et al: Evaluation of the epidermal growth factor receptor (EGFR) in colorectal tumours and lymph node metastases. Eur J Cancer 38: 2258-2264, 2002.

12. Spano JP, Lagorce C, Atlan D, et al: Impact of EGFR expression on colorectal cancer patient prognosis and survival. Ann Oncol 16: 102-108, 2005.

13. Galizia G, Lieto E, Ferraraccio F, et al: Prognostic significance of epidermal growth factor receptor expression in colon cancer patients undergoing curative surgery. Ann Surg Oncol 13: 823-835, 2006

14. Resnic MB, Routhier J, Konkin T, Sabo E and Pricolo VE: Epidermal growth factor receptor, c-MET, beta-catenin, and p53 expression as prognostic indicators in stage II colon cancer: a tissue microarray study. Clin Cancer Res 10: 3069-3075, 2004.

15. Iacopetta B: Are there two sites to colorectal cancer? Int J Cancer 101: 403-408, 2002.

16. Sugai T, Habano W, Jiao YF, Tsukahara M, Takeda Y, Otsuka K and Nakamura S: Analysis of molecular alterations in left- and right- sided colorectal carcinomas reveals distinct pathways of carcinogenesis. J Mol Diagn 8: 193-201, 2006.

17. Nawa T, Kato J, Kawamoto H, et al: Differences between right and left-sided colon cancer in patient characteristics, cancer morphology and histology. J Gastroenderol Hepatol 23: 418-423, 2008.

18. Papagiorgis PC,Zizi AE, Tseleni S, et al: Site impact on colorectal cancer biological behavior in terms of clinicopathological and molecular features. J BUON 16: 84-92, 2011.

19. Menguid R, Slidell MB, Wolfgang L, Chang DC and Ahuja N: Is there a difference in survival between right- versus left-sided colon cancers? Ann Surg Oncol 15: 2388-2394, 2008. 
20. Elsaleh H, Joseph D, Grieu F, Zeps N, Spry N and Iacopetta B Association of tumour site and sex with survival benefit from adjuvant chemotherapy in CRC. Lancet 355: 1745-1750, 2000.

21. Zlobec I, Steele R, Michel R, Compton C, Lugli A and Jass J: Scoring of p53, VEGF, Bcl-2 and APAF-1 immunohistochemistry and interobserver reliability in colorectal cancer. Mod Pathol 19: 1236-1242, 2006.

22. Lee JC, Wang ST, Chow NH and Yang HB: Investigation of the prognostic value of coexpressed erbB family members for the survival of colorectal cancer patients after curative surgery. Eur J Cancer 38: 1065-1071, 2002.

23. Takeuchi K, Kuwano H, Tsuzuki Y, Ando T, Sekihara M, Hara T and Asao T: Clinicopathological characteristics of poorly differentiated adenocarcinoma of the colon and rectum. Hepatogastroenterology 51: 1698-1702, 2004.

24. Russo A, Bazan V, Iacopetta D, Kerr D, Soussi T and Gebbia N: The TP53 colorectal cancer international collaborative study on the prognostic and predictive significance of p53 mutation: influence of tumor site, type of mutation, and adjuvant treatment. J Clin Oncol 23: 7518-7528, 2005.

25. Kastrinakis WV, Ramchurren N, Rieger KM, Hest DT, Loda M, Steel G and Summerhayes IC: Increased incidence of p53 mutations is associated with hepatic metastasis in colorectal neoplastic progression. Oncogene 11: 647-652, 1995

26. Oden-Gangloff A, Di Fiore F, Bibeau F, et al: TP53 mutations predict disease control in metastatic colorectal cancer treated with cetuximab-based chemotherapy. Br J Cancer 100: $1330-1335,2009$

27. Chun P and Wainberg A: Adjuvant chemotherapy for stage II colon cancer: the role of molecular markers in choosing therapy. Gastrointest Cancer Res 3: 191-196, 2009.

28. O'Connell MJ, Lavery I, Yothers G, et al: Relationship between tumor gene expression and recurrence in four independent studies of patients with stage II/III colon cancer treated with surgery alone or surgery plus adjuvant fluorouracil plus leucovorin. J Clin Oncol 28: 3937-3944, 2010.

29. Bacolod MD and Barany F: Molecular profiling of colon tumors: the search for clinically relevant biomarkers of progression, prognosis, therapeutics, and predisposition. Ann Surg Oncol, 2011, doi: $10.1245 / \mathrm{s} 10434-011-1615-5$.
30. Allegra CJ, Jessup JF, Somerfield MR, et al: American Society of Clinical Oncology provisional clinical opinion: testing for KRAS gene mutations in patients with metastatic colorectal carcinoma to predict response to anti-epidermal growth factor receptor monoclonal antibody therapy. J Clin Oncol 27: 2091-2096, 2009.

31. Laurent-Puig P, Cayre A, Manceau G, et al: Analysis of PTEN BRAF, and EGFR status in determining benefit from cetuximab therapy in wild-type KRAS metastatic colon cancer. J Clin Oncol 27: 5924-5930, 2009.

32. Elnatan J, Coh HS and Smith DR: C-KI-RAS activation and the biological behaviour of proximal and distal colonic adenocarcinomas. Eur J Cancer 32A: 491-497, 1996.

33. Chang MH, Lee IK, Si Y, Lee KS, Woo IS and Byun JH: Clinical impact of K-ras mutation in colorectal cancer patients treated with adjuvant FOLFOX. Cancer Chemother Pharmacol 68: 317-323, 2011.

34. Dukes C and Bussey H: The spread of rectal cancer and its effect on prognosis. Br J Cancer 12: 309-320, 1958

35. Atkins D, Reiffen KA, Tegtmeier CL, Winther H, Bonato MS and Störkel S: Immunohistochemical detection of EGFR in paraffin-embedded tumor tissue: variation in staining intensity due to choice of fixative and storage time of tissue sections. J Histochem Cytochem 58: 893-901, 2004.

36. Tampellini M, Longo M, Cappia S, et al: Co-expression of EGFR receptor, TGFa and S6 kinase is significantly associated with colorectal carcinomas with distant metastases at diagnosis. Virchows Arch 450: 321-328, 2007.

37. Rigopoulos DN, Tsiambas E, Lazaris AC, et al: Deregulation of EGFR/VEGF/HIF-1a signaling pathway in colon adenocarcinoma based on tissue microarrays analysis. J BUON 15: 107-115, 2010.

38. Messa C, Russo F, Caruso MG and Di Leo A: EGF, TGF-alpha, and EGF-R in human colorectal adenocarcinoma. Acta Oncol 37: 285-289, 1998.

39. Benedix F, Schmidt U, Mroczkowski P, Gastinger I, Lippert H and Kube R: Colon carcinoma - classification into right and left sided cancer or according to colonic subsite? - Analysis of 29,568 patients. Eur J Surg Oncol 37: 134-139, 2011. 